Kinder- und Jugendpsychiatrie

\title{
Fischöl in der Prävention
}

\author{
Omega 3-Fettsäuren können den Ausbruch von Psychosen verhindern bzw. verzögern.
}

Die Behandlung mit Fischölkapseln mit mehrfach ungesättigten Omega 3-Fettsäuren könnte bei Jugendlichen und jüngeren Erwachsenen den Ausbruch von Psychosen verhindern. Das ist das Ergebnis einer Studie an der Universitätsklinik für Kinder- und Jugendpsychiatrie der MedUni Wien in Kooperation mit Orygen - The National Centre of Excellence in Youth Mental Health der Universität Melbourne, die nun im Top-Journal „Nature Communications" veröffentlicht wurde.

Die nun veröffentlichten Resultate basieren auf den Ergebnissen einer Studie aus dem Jahr 2010, als an der Universitätsklinik für Kinder- und Jugendpsychiatrie der MedUniWien festgestellt wurde, dass die Gabe von Fischölkapseln mit mehrfach ungesättigten Omega 3-Fettsäuren das Risiko, an einer Psychose zu erkranken, über den Zeitraum eines Jahres um 22 Prozent senkt.

Nun liegen die Daten des Follow-Ups bei 71 Patienten zwischen 13 und 25 Jahren über einen Zeitraum von 6,7 Jahren vor.,Es hat sich gezeigt, dass die Langzeitwirkung einen doppelten Effekt zeigt: Einerseits kann das Risiko, eine Psychose zu bekommen, deutlich gesenkt werden, andererseits lässt sich der Ausbruch der Psychose mit Gabe des Fischöls verzögern", erklären die Studienautoren. In der Gruppe, die die hoch dosierten Omega 3-Fettsäuren erhielt, sank das Risiko für das Auftreten einer Psychose auf 9,8 Prozent. In der Placebogruppe lag der Wert bei 40 Prozent.

Die nun veröffentlichten Ergebnisse stärken die Hoffnung, so die Forscher, mit einer natürlichen Substanz künftig den Ausbruch psychotischer Störungen bei Risikogruppen auch längerfristig verzögern und möglicherweise verhindern zu können. Dazu müssen die Resultate aber in einer zweiten Studie bestätigt werden. Diese läuft bereits. Die MedUni Wien ist wieder maßgeblich daran beteiligt.
Quelle: Pressemeldung der MedUniWien, 11.8.2015

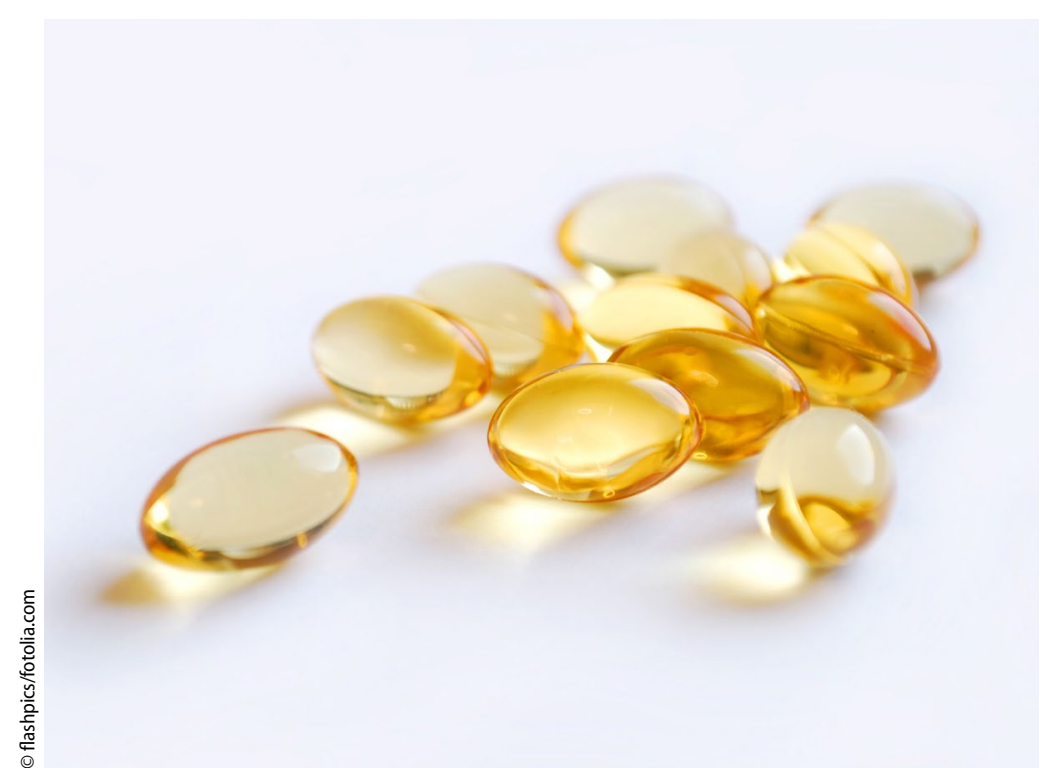

$\Delta$ Die vorliegende Studie befasst sich mit der Gabe von Fischöl (in Kapseln - siehe Foto) zur Prävention psychotischer Erkrankungen

\section{Zwei bis drei Prozent der} Bevölkerung leiden an Psychosen Psychotische Erkrankungen betreffen mindestens zwei bis drei Prozent der Bevölkerung. Sie treten meist beim Jugendlichen oder jungen Erwachsenen erstmals auf, verlaufen dann aber oft chronisch und haben schwerwiegende Auswirkungen für die Betroffenen und ihre Familien. Präventive Behandlungsansätze sind derzeit noch wenig erforscht. Dabei gibt es Vorzeichen, die es ermöglichen, Patienten mit einem hohen Risiko für psychotische Störungen zu identifizieren. Eine Psychose tritt nämlich nur ganz selten plötzlich und "wie aus dem Nichts" auf; in den meisten Fällen bestehen bereits über Wochen oder Monate Symptome in abgeschwächter Form. In dieser so genannten Prodromalphase setzen die Forschungsarbeiten rund um die Behandlung mit Fischölkapseln an.

\section{Fünf Forschungscluster an der MedUni Wien}

Insgesamt sind fünf Forschungscluster der MedUni Wien etabliert. Dort werden in der Grundlagen- wie in der klinischen Forschung vermehrt Schwerpunkte an der MedUni Wien gesetzt. Die Forschungscluster umfassen medizinische Bildgebung, Krebsforschung/Onkologie, kardiovaskuläre Medizin, medizinische Neurowissenschaften und Immunologie. Die vorliegende Arbeit fällt in den Bereich des Clusters für medizinische Neurowissenschaften.

Pädiatrie \& Pädologie DOI 10.1007/s00608-015-0313-0

๑) Springer-Verlag Wien 2015 